\title{
The rotag library: generating protein structure-specific side-chain rotamer libraries
}

\author{
A. Grybauskas, S. Gražulis \\ Vilnius University Life Sciences Center, Sauletekio al. 7, 10257 Vilnius \\ algirdas.grybauskas@gmc.vu.lt
}

Identifying the probable positions of the protein side-chains is one of the protein modelling steps that can greatly improve the prediction of protein-ligand, protein-protein interactions. With some exceptions, most of the strategies predicting the side-chain conformations use predetermined angles, also called rotamer libraries, that are usually generated from the subset of high-quality protein structures. Although, these libraries are very useful when selecting possible side-chain atom positions, the overall validity and usability regarding specific protein structure should be studied further.

In order to get well-rounded rotamer library, there should be the balance between the coverage and the quantity of possible side-chain positions. The lack of possible side-chain rotamers will hinder the correct selection of atom positions and the over abundance - the fast selection for protein structure predictions.

We are suggesting the approach that would cover both the coverage and the accuracy of the rotamer library. The rotag software was developed in order to accommodate both these problems. It scans side-chain conformations using dead-end elimination strategy and evaluating potential energies on each calculation step.

The additional challenge that we faced was to have proper method to compare rotamer libraries. The best-case RMSD, best-case dihedral angles and average rotamer choice parameters were selected as good candidates for the comparisons. Multiple rotamer libraries were compared: Dunbrack, Dynameomics, Penultimate and those generated with rotag.

The comparisons revealed that the rotamer libraries that were created from the subset of existing protein structures sometimes lack rotamer positions for certain side-chains of the target proteins. Using more flexible methods, such as rotag, increases the probability of the inclusion of correct conformations. However, not in all cases these flexible methods produce the correct subset of potential candidates.

Keywords: rotamer; side-chain 